\title{
Roboticized Reform for Yuchai YC13-8 Hydraulic Excavator ${ }^{1}$
}

\author{
Yukun $\mathrm{Li}^{1, \mathrm{a}^{*}}$, Xiaoping Liu ${ }^{1, \mathrm{~b}}$ and Jihong Guan ${ }^{1, \mathrm{c}}$ \\ ${ }^{1}$ College of Mechanical Engineering, Yanshan University, Hebei Province, 066004, China \\ alyk@ysu.edu.cn, b1096272068@qq.com, '1553232477@qq.com
}

\begin{abstract}
Keywords: excavator, control system transformation, trajectory control, EPEC
Abstract: This paper points out the meaning of the electronic control transformation of excavator system. Based on the analysis of the YC13-8 hydraulic control system, we put forward the technological scheme of YC13-8 excavator electronic control transformation, and introduce the composition of control system and the method of intelligent trajectory control after the reforming. The experimental results show that the transformation scheme is not only simple and economic, but also of great practicability and reliability.
\end{abstract}

\section{Introduction}

Hydraulic excavator is one of the engineering machinery with most typical function, most complex structure and most widely application. As the mainstream of engineering machinery products, which plays a very important role in the industry and civil construction, transportation, water resources and electric power engineering, mining engineering, and military engineering, etc [1,2]. However, with the expansion of excavator working field, some harmful, bad, dangerous work environment, such as landslides, explosions, chemical environment and space development, etc, is not suitable for driver operation. Therefore, in order to realize the automatic operations of excavator, it is very important to reform the existing excavator control system. What's more, the key of achieving the goal of automatic excavation is the ability of placing the bucket in one specific location and orientation. Therefore, it is also necessary to research the trajectory planning system of the excavator.

\section{Excavator System Introduction}

The paper is mainly about the electronic control transformation of Yuchai YC13-8 small excavator. Yuchai YC13-8 small excavator mainly consists of two parts, and the main actuator is composed of working mechanism, slewing mechanism and walking mechanism. The working mechanism is mainly located in the upper group, which includes the boom cylinder, the stick cylinder and the bucket cylinder, and with mutual coordination of these parts, excavation can be done; the upper group is connected to the low group by a rotary device, which completes the relative rotary movement of the upper group around the low group; The low group mainly includes walking motor and bulldozing device, The hydraulic system drives walking motor to realize movement and the steering of the machine. Bulldozing device may play a role in eliminating obstacles in front, cleaning road and fixing body. Yuchai YC13-8 small excavator has advantages of small volume, high flexibility, strong power, etc, which is suitable for the numerical control transformation. YC13-8 hydraulic excavator system group is shown in figure 1. 


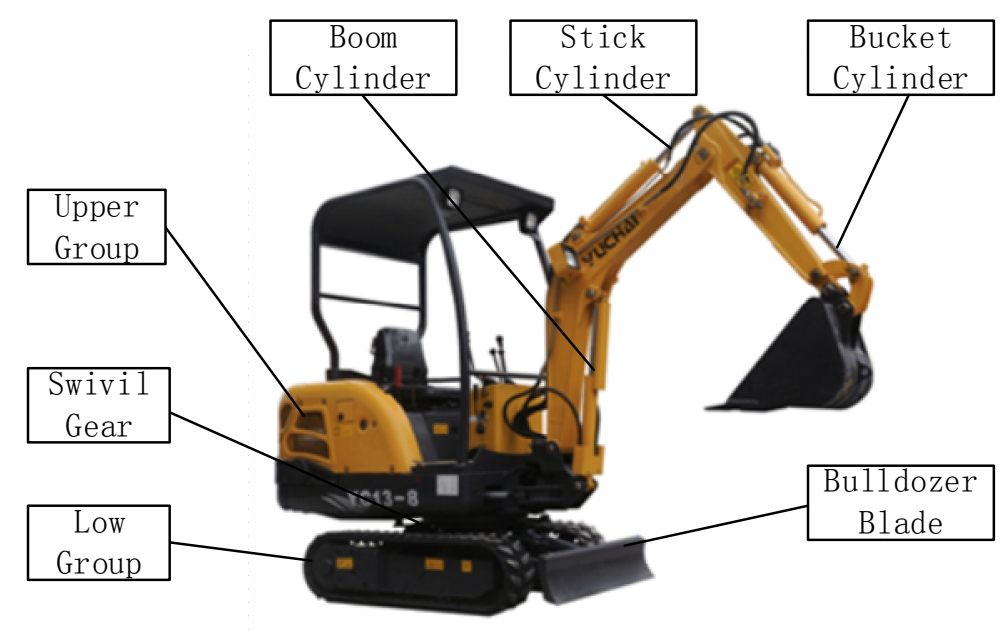

Figure 1 YC13-8 hydraulic excavator system architecture figure

\section{Hydraulic System Reform Scheme}

Yuchai YC13-8 excavator control system adopts full hydraulic pressure drive, quantitative pump throttle control, mechanical lever manipulation. Manipulate the guide handle to change the opening of the pilot valve, so as to change the output pressure of the pilot valve, thus to control the opening of the main valve, and ultimately achieve the purpose of controlling the hydraulic working device, which is characterized in simple structure, stable performance, flexible operation, etc. The excavator control principle before transformation is shown in figure 2.

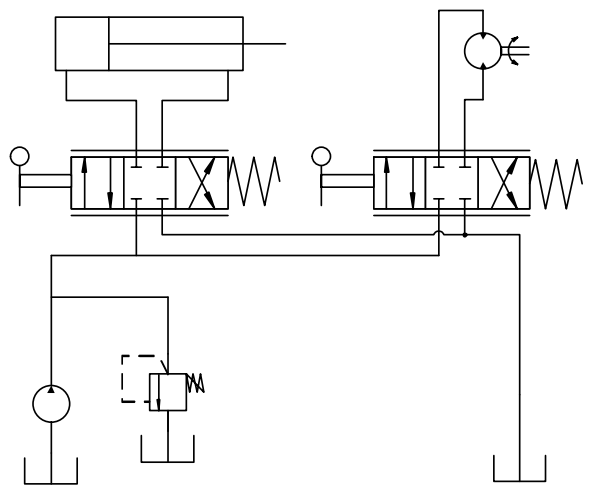

Figure 2 the excavator control principle figure before transformation

In order to realize the YC13-8 excavator electric control transformation, we replace multi-valve and pilot control valve of the original control actuator with multiple parallel electromagnetic directional valve, and add electric proportional throttle valve to control the run speed. Excavator control is based on the motion displacement control of the working device, in order to detect the motion displacement of each hydraulic cylinder, we need to install the cable-displacement sensor in the arm cylinder, the boom cylinder, and the bucket cylinder; In order to detect the operation signal, we can install pressure sensors in the hydraulic cylinder inlet and outlet; It is equipped with wireless operating handle to achieve the remote control of excavator. The excavator hydraulic system principle after transformation is shown in figure 3, proportional cartridge valve blocks is shown in figure 4. 


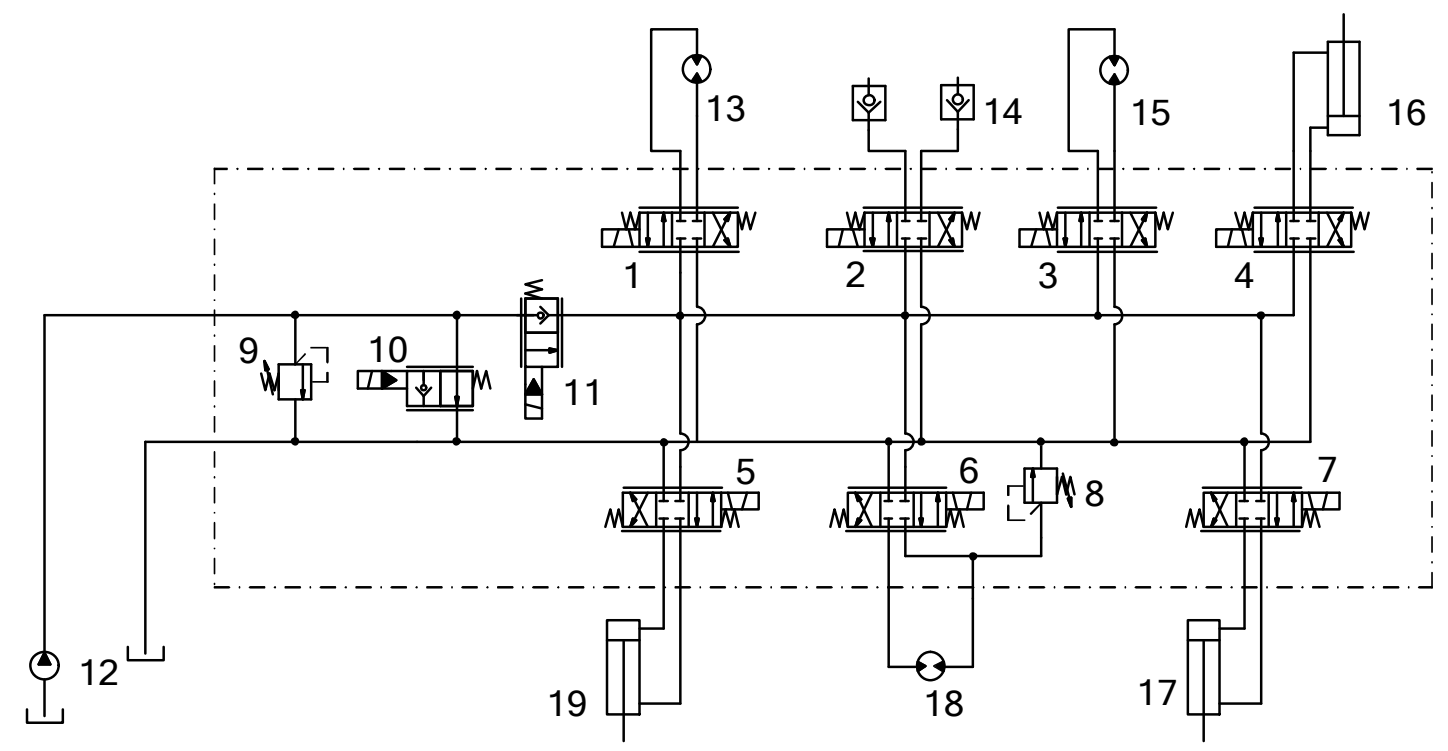

1-7: electromagnetic proportional direction valve; 8-9: regulating valve; 10-11: electromagnetic proportion throttle; 12: hydraulic source: 13: Left travel motor; 14: Alternate joint; 15: Right travel motor; 16: Boom cylinder; 17: Bucket cylinder; 18: Swivel motor; 19: Stick cylinder.

Figure 3 the principle figure of hydraulic system after transformation

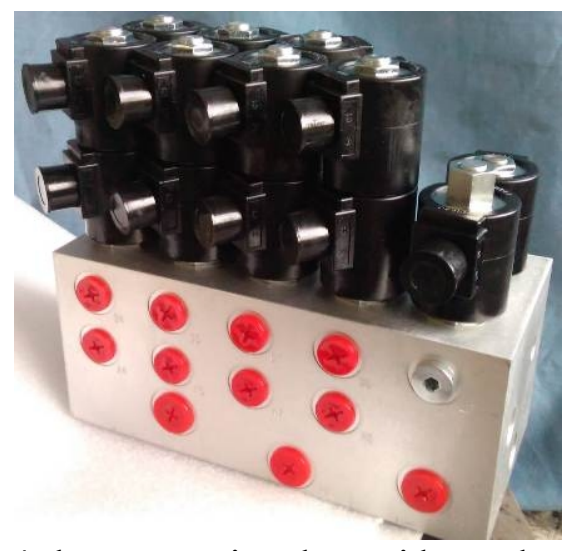

Figure 4 the proportional cartridge valve blocks

Each actuator velocity is jointly regulated by the proportion throttle 10 and 11 . The regulating valve 9 is used to limit the highest pressure, the regulating valve 8 is used to limit the rotating loop pressure. The regulating valve 9 and proportion throttle 11 constitute the inlet speed adjusting loop, and proportion throttle 10 constitute the bypass throttle modulating velocity loop. In practice, we can achieve good load rigidity and high loop efficiency by coordinating the throttle control 10 with 11 .

\section{Composition of the Control System}

The control system consists of the controller, the proportional direction valve, the handle and the displacement and pressure sensor (model as shown in Table 1), which forms a closed loop control. The control module of the system uses SPT-K-2024 series CAN bus control module of EPECOY made in Finland. The EPEC control module is of high security, high performance microprocessor based on long-term bad working conditions, such as high vibration, high temperature and humid environment. It is very suitable for the engineering machinery to control automatically, which sets programmable controller, proportional amplifier, analog input and output module, relay and other functions into one part. The EPEC controller has a large number of input and output ports, it can act as a multifunctional controller to drive the actuator, such as proportional direction valve, as well as an independent controller. 
Table 1.The selection table of control system component

\begin{tabular}{|c|c|}
\hline Item & Version \\
\hline Controller & EPEC2024 \\
\hline $\begin{array}{c}\text { Electromagnetic } \\
\text { proportion valve }\end{array}$ & TS10-36A-0-N-24DG \\
\hline Displacement sensor & GWS250 \\
\hline Pressure sensor & DMP333 \\
\hline Joystick & JCP2 \\
\hline
\end{tabular}

working principle:

The core of excavator control system is the EPEC2024 controller, which is responsible for dealing with all the information. The sensors mainly include cable-displacement sensors and pressure sensors, they respectively detect the displacement of the working device and the press of the driving hydraulic cylinder, and transfer the information to the controller through the $\mathrm{A} / \mathrm{D}$ conversion. The EPEC controller receives a given input from the operating handle, solutes it through internal preset inverse solution algorithm, achieves the corresponding displacement of each hydraulic cylinder, and compares the displacement with the actual displacement which is transferred back through the CAN bus. The output control voltage is ascertained, based on the size, plus or minus of the error, the controller produces PWM signals to drive the proportional direction valve, making the pressure oil flow into the hydraulic cylinder, and drive the working device to perform the action. The output signal control the corresponding proportional direction valve to control the output, whose purpose is to coordinate the arm cylinder, the boom cylinder, and the bucket cylinder, and make the bucket cylinder excavate automatically in accordance with prescribed trajectory. The hardware structure of working device control system [3] is shown in figure 5.

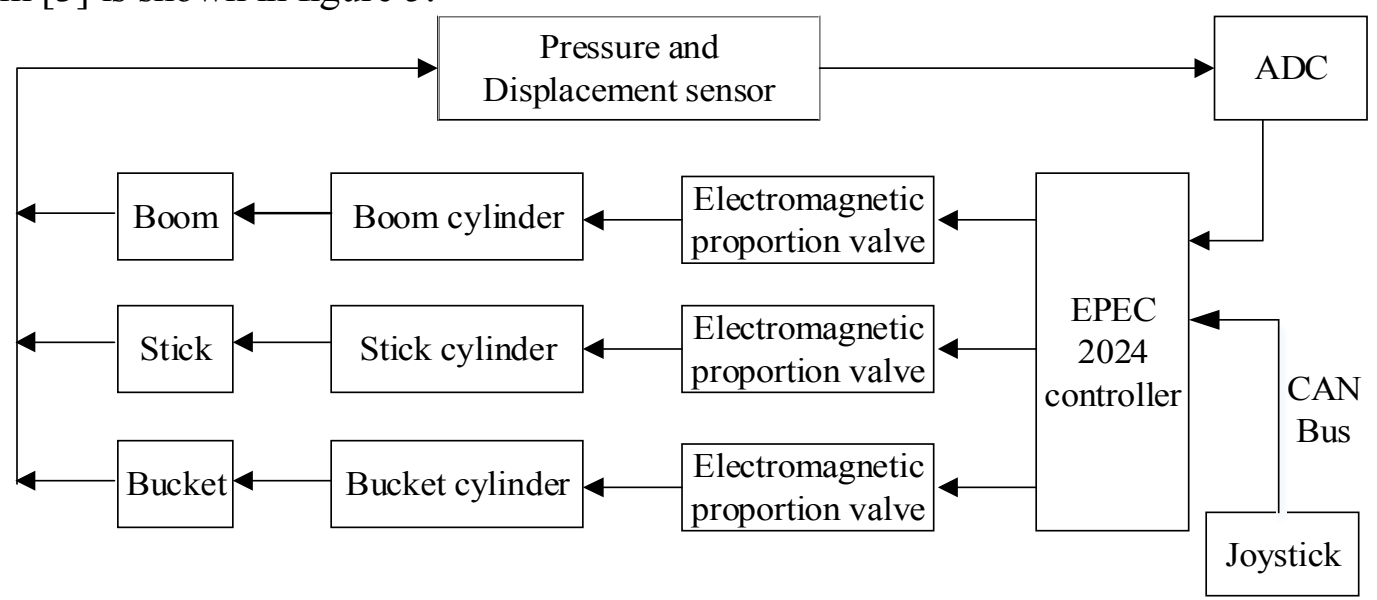

Figure 5 the hardware structure figure of working device control system

\section{The Intelligent Trajectory Control}

The Position Description of Bucket Tooth

The position of bucket tooth in the base coordinate system (X, Y, Z) and the attitude angle of bucket (bucket tooth and bucket hinge point on the surface of the attachment at angle) can only determine the space state of working mechanism. Excavator working device consists of the arm, the boom, the bucket, the super-structure, etc, and can be seen as three degrees of freedom system without thinking about rotating. Therefore, the geometry position of bucket depends on the length of the arm cylinder, the boom cylinder and the bucket cylinder, thus, we can control the position of bucket tooth by controlling the moving length of each hydraulic cylinder. The paper choose the hydraulic cylinder length (L1, L2, L3) of working device as the control variables, as shown in figure 6, the displacement sensor feed back the changes of the hydraulic cylinder displacement to the controller, which can correct action in real time by control algorithm, and improve the control precision. 


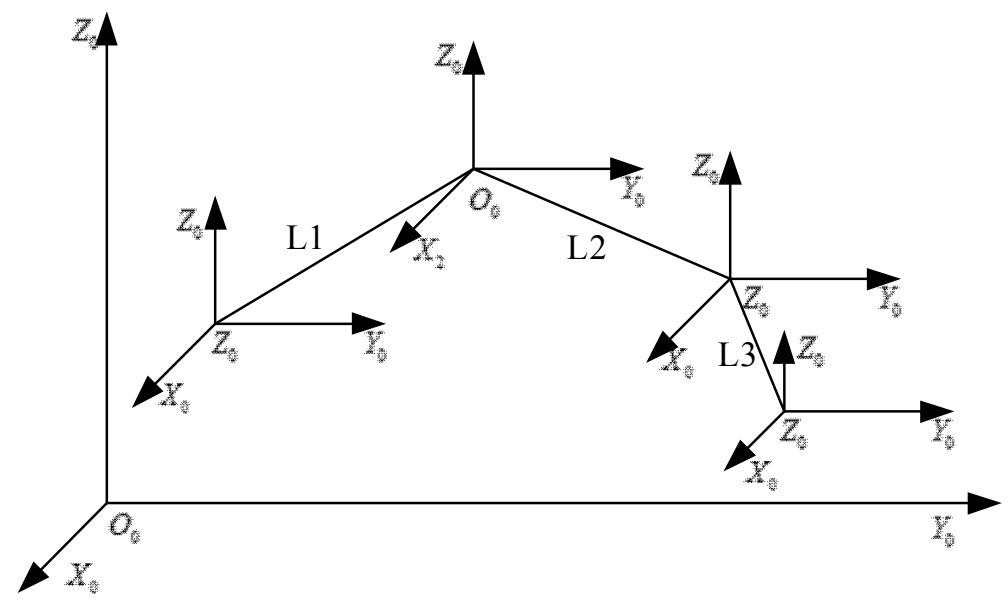

Figure 6 the D-H coordinate of the excavator working device

The PID Control Algorithm

The PID control algorithm can meet the general control requirements for its easy parameters tuning and flexible structure. Its outstanding advantage is that it is not required to know the mathematical model of the controlled object, and that it has strong robustness to the system parameter variation. Figure 6 is a PID control system of single loop. PID control algorithm uses the location algorithm.

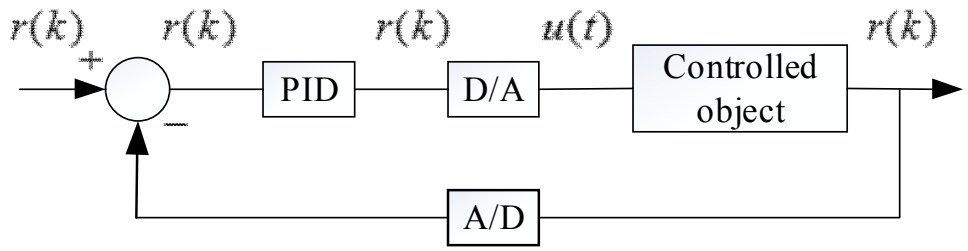

Figure 7 the PID control system of single loop.

$u(k)=k_{p}\left\{e(k)+T_{s} / T_{i} \sum_{j=0}^{k} e(j)+T_{d} / T_{s}[e(k)-e(k-1)]\right\}+u_{0}$

$u(k)$ - the control of the first $\mathrm{k}$ sampling time

$u_{0}$ - the base value of control value

$k_{p}$ - the scaling factor

$T_{s}$ - the sampling period

$T_{i}$ - the integral time

$T_{d}$ - the derivative time

The Control Algorithm

The intelligent trajectory control of excavator can achieve the horizontal excavation, slope excavation, control of digging depth and other functions [4]. In the process of automatic excavation, the planned trajectory is in configuration space, namely in the natural coordinate system, and the control is implemented in the drive space. To obtain the control signal, the preset trajectory based on a certain step is divided into several parts, thus we can get a group of bucket teeth configuration coordinate sequence. According to the bucket configuration sequence and the transformation matrix [5], we can obtain the length sequences of the oil cylinders of the corresponding bars, and implement the preset trajectory with the PID algorithm, and then complete the preset trajectory. The program flow chart of path implementation is shown in figure 8 . 


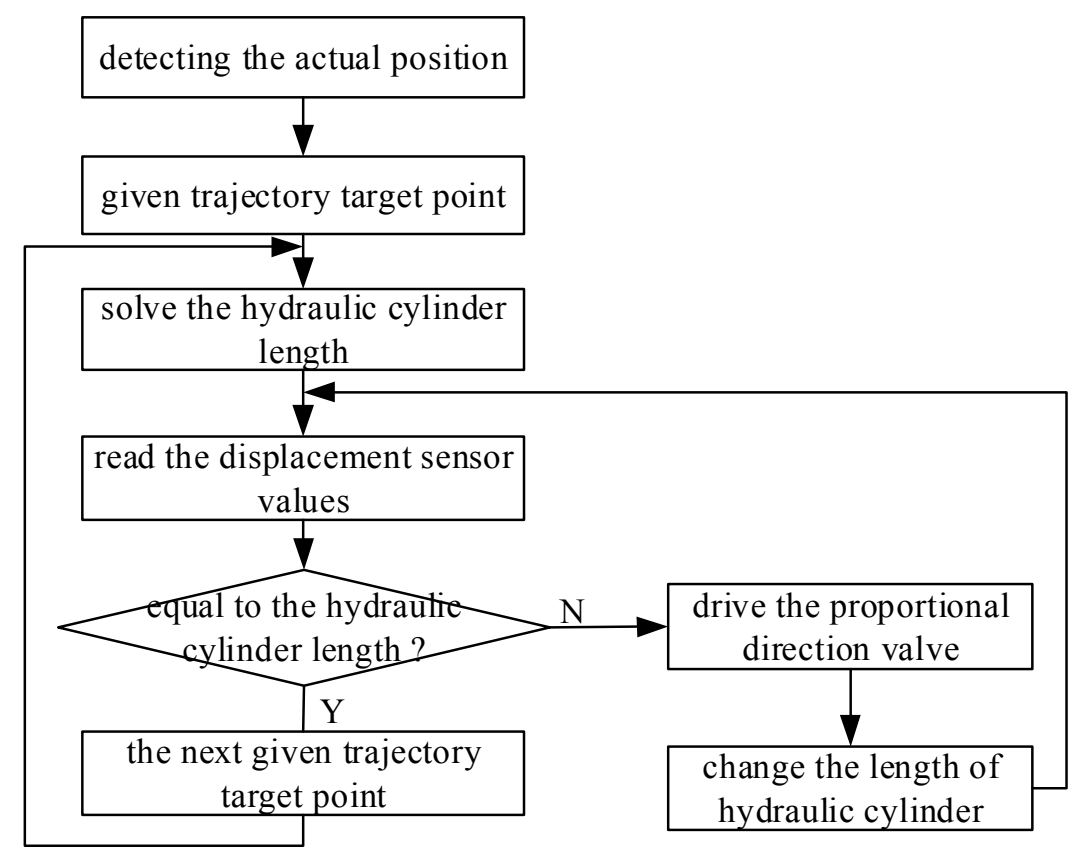

Figure 8 the program flow chart of path implementation

\section{Summary}

In this paper, the YuChai YC13-8 small excavator hydraulic system is reformed, and configured with corresponding electronic control system. The improved excavator can meet the requirements of mining operations, and can adapt to the harsh working environment of engineering machinery; The improved system is less dependent on the operators' technical requirements and can relieve the work intensity greatly. The improved scheme is simple and economic, practical and reliable, which provides a feasible scheme for electronic control system of hydraulic excavator and the excavator electronic control transformation.

\section{References}

[1] Yang Zhigang, Gu Yejun. Introduction of operation practice of hydraulic excavator[J]. Science \& Technology Information, 2012(29).

[2] Feng Peien. The construction machinery automation[J]. Construction Machinery and Equipment, 1989(20): 20 22

[3] Guo Gang, Gong Liehang. Electro-hydraulic proportional technology in the application of the excavator[J]. Construction Machinery Technology \& Management, 1998, 06, 010

[4] Hu Chong, Li Zhi, Xu Chuan. A CAN bus based trajectory planning system of excavator[J]. Construction Machinery and Equipment, 2006 (7)

[5] Zhang Haitao. Research of robot motion control system of the working device of hydraulic excavator[D]. Changsha: Central South University. 2004, 21-31. 